\title{
Sharp bounds for the Neuman mean in terms of the quadratic and second Seiffert means
}

\author{
Yu-Ming Chu ${ }^{1 *}$, Hua Wang ${ }^{2}$ and Tie-Hong Zhao ${ }^{3}$
}

"Correspondence:

chuyuming2005@126.com

'School of Mathematics and

Computation Science, Hunan City

University, Yiyang, 413000, China

Full list of author information is

available at the end of the article

\section{Abstract}

In this paper, we prove that $\alpha=0$ and $\beta=\frac{\sqrt{3} \pi-4 \log (2+\sqrt{3})}{(\sqrt{2} \pi-4) \log (2+\sqrt{3})}=0.29758 \cdots$ are the best possible constants such that the double inequality

$$
\alpha Q(a, b)+(1-\alpha) T(a, b)<S_{C A}(a, b)<\beta Q(a, b)+(1-\beta) T(a, b)
$$

holds for all $a, b>0$ with $a \neq b$, where $Q(a, b)=\sqrt{\left(a^{2}+b^{2}\right) / 2}$,

$$
S_{C A}(a, b)=\frac{(a-b) \sqrt{3\left(a^{2}+b^{2}\right)+2 a b}}{2(a+b) \sinh ^{-1}\left(\frac{(a-b) \sqrt{3\left(a^{2}+b^{2}\right)+2 a b}}{(a+b)^{2}}\right)}
$$

and $T(a, b)=(a-b) /[2 \arctan ((a-b) /(a+b))]$ are the quadratic, Neuman and second Seiffert means of $a$ and $b$, respectively.

MSC: $26 \mathrm{E} 60$

Keywords: Neuman mean; quadratic mean; second Seiffert mean

\section{Introduction}

For $a, b>0$ with $a \neq b$, the Neuman mean $S_{C A}(a, b)[1,2]$ derived from the SchwabBorchardt mean $[3,4]$, the quadratic mean $Q(a, b)$ and the second Seiffert mean $T(a, b)$ [5] are given by

$$
\begin{aligned}
& S_{C A}(a, b)=\frac{(a-b) \sqrt{3\left(a^{2}+b^{2}\right)+2 a b}}{2(a+b) \sinh ^{-1}\left(\frac{(a-b) \sqrt{3\left(a^{2}+b^{2}\right)+2 a b}}{(a+b)^{2}}\right)}, \\
& Q(a, b)=\sqrt{\frac{a^{2}+b^{2}}{2}}
\end{aligned}
$$

and

$$
T(a, b)=\frac{a-b}{2 \arctan \left(\frac{a-b}{a+b}\right)},
$$

respectively, where $\sinh ^{-1}(x)=\log \left(x+\sqrt{1+x^{2}}\right)$ is the inverse hyperbolic sine function. Recently, the Neuman, quadratic and second Seiffert means have been the subject of intensive

○2014 Chu et al.; licensee Springer. This is an Open Access article distributed under the terms of the Creative Commons Attribution License (http://creativecommons.org/licenses/by/2.0), which permits unrestricted use, distribution, and reproduction in any medium, provided the original work is properly cited. 
research. In particular, many remarkable inequalities for these means can be found in the literature [1-4, 6-15].

Let $A(a, b)=(a+b) / 2$ and $C(a, b)=\left(a^{2}+b^{2}\right) /(a+b)$ be the arithmetic and contraharmonic means of $a$ and $b$, respectively. Then Neuman [1] proved that the inequalities

$$
A(a, b)<T(a, b)<S_{C A}(a, b)<Q(a, b)<C(a, b)
$$

hold for any $a, b>0$ with $a \neq b$.

In $[1,2]$, Neuman found that $\alpha_{1}=[\sqrt{3}-\log (2+\sqrt{3})] / \log (2+\sqrt{3})=0.315 \cdots, \beta_{1}=1 / 3$, $\alpha_{2}=1 / 3, \beta_{2}=[\log 3-2 \log (\log (2+\sqrt{3}))] /(2 \log 2)=0.395 \cdots, \alpha_{3}=2 \log (2+\sqrt{3}) / 3-1=$ $0.520 \cdots$ and $\beta_{3}=2 / 3$ are the best possible constants such that the double inequalities

$$
\begin{aligned}
& \alpha_{1} C(a, b)+\left(1-\alpha_{1}\right) A(a, b)<S_{C A}(a, b)<\beta_{1} C(a, b)+\left(1-\beta_{1}\right) A(a, b), \\
& C^{\alpha_{2}}(a, b) A^{1-\alpha_{2}}(a, b)<S_{C A}(a, b)<C^{\beta_{2}}(a, b) A^{1-\beta_{2}}(a, b)
\end{aligned}
$$

and

$$
\frac{\alpha_{3}}{A(a, b)}+\frac{1-\alpha_{3}}{C(a, b)}<\frac{1}{S_{C A}(a, b)}<\frac{\beta_{3}}{A(a, b)}+\frac{1-\beta_{3}}{C(a, b)}
$$

hold for any $a, b>0$ with $a \neq b$.

He et al. [16] proved that $\alpha=1 / 2+\sqrt{\sqrt{3} / \log (2+\sqrt{3})-1} / 2$ and $\beta=1 / 2+\sqrt{3} / 6$ are the best possible constants in $[1 / 2,1]$ such that the double inequality

$$
C[\alpha a+(1-\alpha) b, \alpha b+(1-\alpha) a]<S_{C A}(a, b)<C[\beta a+(1-\beta) b, \beta b+(1-\beta) a]
$$

holds for any $a, b>0$ with $a \neq b$.

In $[17,18]$, the authors proved that the double inequalities

$$
\begin{aligned}
\alpha & {\left[\frac{1}{3} C(a, b)+\frac{2}{3} A(a, b)\right]+(1-\alpha) C^{1 / 3}(a, b) A^{2 / 3}(a, b) } \\
& <S_{C A}(a, b)<\beta\left[\frac{1}{3} C(a, b)+\frac{2}{3} A(a, b)\right]+(1-\beta) C^{1 / 3}(a, b) A^{2 / 3}(a, b)
\end{aligned}
$$

and

$$
\lambda A(a, b)+(1-\lambda) Q(a, b)<S_{C A}(a, b)<\mu A(a, b)+(1-\mu) Q(a, b)
$$

hold for any $a, b>0$ with $a \neq b$ if and only if $\alpha \leq \frac{3[\sqrt[3]{2} \log (2+\sqrt{3})-\sqrt{3}]}{(3 \sqrt[3]{2}-4) \log (2+\sqrt{3})}=0.7528 \cdots, \beta \geq 4 / 5$, $\lambda \geq 1 / 3$ and $\mu \leq \frac{\sqrt{2} \log (2+\sqrt{3})-\sqrt{3}}{(\sqrt{2}-1) \log (2+\sqrt{3})}=0.2390 \cdots$.

The main purpose of this paper is to present the best possible constants $\alpha$ and $\beta$ such that the double inequality

$$
\alpha Q(a, b)+(1-\alpha) T(a, b)<S_{C A}(a, b)<\beta Q(a, b)+(1-\beta) T(a, b)
$$

holds for any $a, b>0$ with $a \neq b$. All numerical computations are carried out using MATHEMATICA software. 


\section{Lemmas}

In order to prove our main results, we need several lemmas, which we present in this section.

Lemma 2.1 The double inequality

$$
-\frac{2 x}{3}+\frac{16 x^{3}}{45}-\frac{2 x^{5}}{7}<\frac{x}{\left(1+x^{2}\right) \arctan ^{2} x}-\frac{1}{\arctan x}<-\frac{2 x}{3}+\frac{16 x^{3}}{45}
$$

holds for $x \in(0,0.6)$.

Proof Let

$$
\begin{aligned}
& \phi_{1}(x)=x-\left(1+x^{2}\right) \arctan x+\left(\frac{2 x}{3}-\frac{16 x^{3}}{45}+\frac{2 x^{5}}{7}\right)\left(1+x^{2}\right) \arctan ^{2} x, \\
& \phi_{2}(x)=x-\left(1+x^{2}\right) \arctan x+\left(\frac{2 x}{3}-\frac{16 x^{3}}{45}\right)\left(1+x^{2}\right) \arctan ^{2} x .
\end{aligned}
$$

Then we only need to show that $\phi_{1}(x)>0$ and $\phi_{2}(x)<0$ for $x \in(0,0.6)$.

Taking the differentiation of $\phi_{1}(x)$ yields

$$
\begin{aligned}
\phi_{1}(0) & =0, \\
\phi_{1}^{\prime}(x) & =\frac{2 \arctan x}{315} \phi_{1}^{*}(x),
\end{aligned}
$$

where

$$
\begin{aligned}
& \phi_{1}^{*}(x)=\left(105+147 x^{2}-55 x^{4}+315 x^{6}\right) \arctan x-x\left(105+112 x^{2}-90 x^{4}\right), \\
& \phi_{1}^{*}(0)=0, \\
& \phi_{1}^{* \prime}(x)=\frac{x}{1+x^{2}} \phi_{1}^{* *}(x),
\end{aligned}
$$

where

$$
\phi_{1}^{* *}(x)=2\left(147+37 x^{2}+835 x^{4}+945 x^{6}\right) \arctan x-x\left(294-59 x^{2}-765 x^{4}\right) .
$$

It is well known that the inequality

$$
\arctan x>x-\frac{x^{3}}{3}
$$

holds for all $x \in(0,1)$.

Equation (2.9) and inequality (2.10) lead to the conclusion that

$$
\begin{aligned}
\phi_{1}^{* *}(x) & >2\left(147+37 x^{2}+835 x^{4}+945 x^{6}\right)\left(x-\frac{x^{3}}{3}\right)-x\left(294-59 x^{2}-765 x^{4}\right) \\
& =\frac{x^{3}}{3}\left[105+7,231 x^{2}+2,110 x^{4}+1,890 x^{4}\left(1-x^{2}\right)\right]>0
\end{aligned}
$$

for $x \in(0,0.6)$. 
Therefore, $\phi_{1}(x)>0$ for $x \in(0,0.6)$ follows easily from (2.4)-(2.8) and (2.11).

Differentiating $\phi_{2}(x)$ leads to

$$
\begin{aligned}
& \phi_{2}(0)=0, \\
& \phi_{2}^{\prime}(x)=-\frac{2 \arctan x}{45} \phi_{2}^{*}(x),
\end{aligned}
$$

where

$$
\phi_{2}^{*}(x)=\left(15 x+16 x^{3}\right)-\left(15+21 x^{2}-40 x^{4}\right) \arctan x .
$$

It is well known that the inequality

$$
\arctan x<x-\frac{x^{3}}{3}+\frac{x^{5}}{5}
$$

holds for all $x \in(0,1)$.

Equation (2.14) and inequality (2.15) lead to the conclusion that

$$
\begin{aligned}
\phi_{2}^{*}(x) & >\left(15 x+16 x^{3}\right)-\left(15+21 x^{2}-40 x^{4}\right)\left(x-\frac{x^{3}}{3}+\frac{x^{5}}{5}\right) \\
& =\frac{x^{5}}{15}\left(660-263 x^{2}+120 x^{4}\right)>0
\end{aligned}
$$

for $x \in(0,0.6)$.

Therefore, $\phi_{2}(x)<0$ for $x \in(0,0.6)$ follows from (2.12) and (2.13) together with (2.16).

Lemma 2.2 The double inequality

$$
\frac{x}{\sqrt{1+x^{2}}}+\frac{x}{\left(1+x^{2}\right) \arctan ^{2} x}-\frac{1}{\arctan x}>\frac{x}{3}-\frac{x^{3}}{6}
$$

holds for $x \in(0,0.6)$.

Proof A simple computation leads to

$$
\begin{aligned}
& \left(1-\frac{x^{2}}{2}+\frac{x^{4}}{4}\right)^{2}\left(1+x^{2}\right) \\
& \quad=1-\frac{x^{4}}{16}\left[8\left(\frac{\sqrt{2}}{2}+x\right)\left(\frac{\sqrt{2}}{2}-x\right)+2 x^{4}+x^{4}\left(1-x^{2}\right)\right]<1
\end{aligned}
$$

for $x \in(0,0.6)$. This implies

$$
\frac{x}{\sqrt{1+x^{2}}}>x-\frac{x^{3}}{2}+\frac{x^{5}}{4}
$$

for $x \in(0,0.6)$. 
From Lemma 2.1 and (2.18) we clearly see that

$$
\begin{aligned}
& \frac{x}{\sqrt{1+x^{2}}}+\frac{x}{\left(1+x^{2}\right) \arctan ^{2} x}-\frac{1}{\arctan x} \\
& >\left(x-\frac{x^{3}}{2}+\frac{x^{5}}{4}\right)+\left(-\frac{2 x}{3}+\frac{16 x^{3}}{45}-\frac{2 x^{5}}{7}\right) \\
& \quad=\frac{x}{3}-\frac{13 x^{3}}{90}-\frac{x^{5}}{28}=\frac{x}{3}-\frac{x^{3}}{6}+\frac{x^{3}}{28}\left(\sqrt{\frac{28}{45}}+x\right)\left(\sqrt{\frac{28}{45}}-x\right)>\frac{x}{3}-\frac{x^{3}}{6}
\end{aligned}
$$

for $x \in(0,0.6)$.

Lemma 2.3 The inequality

$$
\frac{x}{\left[\sinh ^{-1}\left(x \sqrt{2+x^{2}}\right)\right]^{2}}-\frac{1+x^{2}}{\sqrt{2+x^{2}} \sinh ^{-1}\left(x \sqrt{2+x^{2}}\right)}>-\frac{x}{3}+\frac{2 x^{3}}{45}-\frac{x^{5}}{63}
$$

holds for $x \in(0,1)$.

Proof Let

$$
\begin{aligned}
\varphi(x)= & x \sqrt{2+x^{2}}-\left(1+x^{2}\right) \sinh ^{-1}\left(x \sqrt{2+x^{2}}\right) \\
& +\left(\frac{x}{3}-\frac{2 x^{3}}{45}+\frac{x^{5}}{63}\right)\left[\sinh ^{-1}\left(x \sqrt{2+x^{2}}\right)\right]^{2} \sqrt{2+x^{2}}
\end{aligned}
$$

Then we only need to show that $\varphi(x)>0$ for $x \in(0,1)$.

Differentiating (2.20) leads to

$$
\begin{aligned}
\varphi(0) & =0, \\
\varphi^{\prime}(x) & =\frac{2 x \sinh ^{-1}\left(x \sqrt{2+x^{2}}\right)}{315\left(1+x^{2}\right)} \varphi_{1}(x),
\end{aligned}
$$

where

$$
\begin{aligned}
\varphi_{1}(x)= & -105-133 x^{2}-18 x^{4}+10 x^{6} \\
& +3\left(35+56 x^{2}+20 x^{4}+4 x^{6}+5 x^{8}\right) \frac{\sinh ^{-1}\left(x \sqrt{2+x^{2}}\right)}{x \sqrt{2+x^{2}}} .
\end{aligned}
$$

We claim that

$$
\frac{\sinh ^{-1}\left(x \sqrt{2+x^{2}}\right)}{x \sqrt{2+x^{2}}}>1-\frac{x^{2}}{3}+\frac{2 x^{4}}{15}-\frac{2 x^{6}}{35}
$$

for $x \in(0,1)$. Indeed, let

$$
\omega(x)=\sinh ^{-1}\left(x \sqrt{2+x^{2}}\right)-x \sqrt{2+x^{2}}\left(1-\frac{x^{2}}{3}+\frac{2 x^{4}}{15}-\frac{2 x^{6}}{35}\right),
$$


then $\omega(x)>0$ for $x \in(0,1)$ follows from the fact that

$$
\omega(0)=0, \quad \omega^{\prime}(x)=\frac{16 x^{8}}{35 \sqrt{2+x^{2}}}>0 .
$$

It follows from (2.23) and (2.24) that

$$
\begin{aligned}
\varphi_{1}(x)> & -105-133 x^{2}-18 x^{4}+10 x^{6} \\
& +3\left(35+56 x^{2}+20 x^{4}+4 x^{6}+5 x^{8}\right)\left(1-\frac{x^{2}}{3}+\frac{2 x^{4}}{15}-\frac{2 x^{6}}{35}\right) \\
= & \frac{x^{6}}{35}\left[644+90 x^{2}+16 x^{6}+\left(1-x^{2}\right)\left(239 x^{2}+30 x^{6}\right)\right]>0
\end{aligned}
$$

for $x \in(0,1)$.

Therefore, $\varphi(x)>0$ for $x \in(0,1)$ follows from (2.21) and (2.22) together with (2.25).

Lemma 2.4 The inequality

$$
\arctan x>\frac{\pi}{4}+\frac{x-1}{2}-\frac{2(x-1)^{2}}{7}>\frac{\pi}{4}+\frac{3(x-1)}{4}
$$

holds for $x \in[0.55,1)$.

Proof Let

$$
v(x)=\arctan x-\left[\frac{\pi}{4}+\frac{x-1}{2}-\frac{2(x-1)^{2}}{7}\right] .
$$

Then simple computations lead to

$$
\begin{aligned}
& v(0.55)=0.00030219 \cdots, \quad v(1)=0, \\
& v^{\prime}(x)=\frac{v_{1}(x)}{14\left(1+x^{2}\right)}, \\
& v_{1}(x)=-1+8 x-15 x^{2}+8 x^{3}, \\
& v_{1}(0.55)=0.1935, \quad v_{1}(1)=0, \\
& v_{1}^{\prime}(x)=24\left(x-\frac{15-\sqrt{33}}{24}\right)\left(x-\frac{15+\sqrt{33}}{24}\right) .
\end{aligned}
$$

From $(2.32)$ and $(15-\sqrt{33}) / 24=0.385643 \cdots<0.55$ together with $0.55<(15+$ $\sqrt{33}) / 24=0.864357 \cdots<1$, we clearly see that $v_{1}(x)$ is strictly decreasing on $[0.55,(15+$ $\sqrt{33}) / 24]$ and strictly increasing on $[(15+\sqrt{33}) / 24,1)$. This in conjunction with $(2.31) \mathrm{im}$ plies that there exists $x_{1} \in(0.55,1)$ such that $v_{1}(x)>0$ for $x \in\left[0.55, x_{1}\right)$ and $v_{1}(x)<0$ for $x \in\left(x_{1}, 1\right)$. Then equation (2.29) leads to the conclusion that $v(x)$ is strictly increasing on $\left[0.55, x_{1}\right]$ and strictly decreasing on $\left[x_{1}, 1\right]$. 
Therefore, $v(x)>0$ for $x \in[0.55,1)$ follows from $(2.28)$ and the piecewise monotonicity of $v(x)$. Moreover, the second inequality in (2.26) follows from

$$
\frac{x-1}{2}-\frac{2(x-1)^{2}}{7}>\frac{3(x-1)}{4}+\frac{(1-x)(8 x-1)}{28}>\frac{3(x-1)}{4} .
$$

Lemma 2.5 The inequality

$$
x-\arctan x<\frac{7}{20} x \arctan ^{2} x
$$

holds for $x \in[0.55,1)$.

Proof Let

$$
\mu(x)=x-\arctan x-\frac{7}{20} x \arctan ^{2} x .
$$

Then it suffices to show $\mu(x)<0$ for $x \in[0.55,1)$.

Differentiating $\mu(x)$ yields

$$
\mu^{\prime}(x)=\frac{\mu_{1}(x)}{20\left(1+x^{2}\right)},
$$

where

$$
\mu_{1}(x)=20 x^{2}-14 x \arctan x-7 \arctan ^{2} x-7 x^{2} \arctan ^{2} x .
$$

It is well known that

$$
\arctan x>x-\frac{x^{3}}{3}+\frac{x^{5}}{5}-\frac{x^{7}}{7}
$$

for $x \in(0,1)$.

For $x \in[0.55,0.7]$, it follows from (2.36) and (2.37) that

$$
\begin{aligned}
\mu_{1}(x)< & 20 x^{2}-14 x\left(x-\frac{x^{3}}{3}+\frac{x^{5}}{5}-\frac{x^{7}}{7}\right)-7\left(x-\frac{x^{3}}{3}+\frac{x^{5}}{5}-\frac{x^{7}}{7}\right)^{2} \\
& -7 x^{2}\left(x-\frac{x^{3}}{3}+\frac{x^{5}}{5}-\frac{x^{7}}{7}\right)^{2}=\frac{x^{2}}{1,575} \mu^{*}\left(x^{2}\right)
\end{aligned}
$$

where

$$
\begin{aligned}
\mu^{*}(x)= & -1,575+3,675 x-2,695 x^{2}+2,135 x^{3} \\
& +3,129 x^{4}-861 x^{5}+405 x^{6}-225 x^{7} \\
\mu^{*}(0.49) & =-9.99966 \cdots .
\end{aligned}
$$

Differentiating $\mu^{*}(x)$ yields

$$
\begin{aligned}
\mu^{* \prime}(x)= & \left(3,675-5,390 x+6,405 x^{2}\right)+\left(12,516 x^{3}-4,305 x^{4}\right) \\
& +\left(2,430 x^{5}-1,575 x^{6}\right)>0
\end{aligned}
$$

for $x \in[0.3025,0.49]$. 
Therefore, $\mu^{*}(x)<0$ for $x \in[0.3025,0.49]$ follows from (2.40) and (2.41). This in conjunction with (2.35) and (2.38) implies that $\mu(x)$ is strictly decreasing on $[0.55,0.7]$. Therefore, we get $\mu(x) \leq \mu(0.55)=-0.00151709 \cdots<0$ for $x \in[0.55,0.7]$.

It follows from Lemma 2.4 that

$$
\mu(x)<x-\left[\frac{\pi}{4}+\frac{x-1}{2}-\frac{2(x-1)^{2}}{7}\right]-\frac{7}{20}\left[\frac{\pi}{4}+\frac{x-1}{2}-\frac{2(x-1)^{2}}{7}\right]^{2}=\frac{\mu_{2}(x)}{2,240}
$$

for $x \in(0.7,1)$, where

$$
\begin{aligned}
\mu_{2}(x)= & (1,760-560 \pi)+\left(308 \pi-49 \pi^{2}-644\right) x \\
& +(1,960-420 \pi) x^{2}+(112 \pi-1,252) x^{3}+480 x^{4}-64 x^{5} .
\end{aligned}
$$

Differentiating $\mu_{2}(x)$ yields

$$
\begin{aligned}
& \mu_{2}(0.7)=-1.68877 \cdots, \quad \mu_{2}(1)=-2.9025 \cdots, \\
& \mu_{2}^{\prime}(x)=\left(-644+308 \pi-49 \pi^{2}\right)+(3,920-840 \pi) x+(336 \pi-3,756) x^{2} \\
&+1,920 x^{3}-320 x^{4}, \\
& \mu_{2}^{\prime}(0.7)=-4.73674 \cdots, \quad \mu_{2}^{\prime}(1)=20.6372 \cdots, \\
& \mu_{2}^{\prime \prime}(x)=8\left(490-105 \pi-939 x+84 \pi x+720 x^{2}-160 x^{3}\right), \\
& \mu_{2}^{\prime \prime}(0.7)=-116.173 \cdots, \quad \mu_{2}^{\prime \prime}(1)=360.212 \cdots, \\
& \mu_{2}^{\prime \prime \prime}(x)=24\left(28 \pi-313+480 x-160 x^{2}\right) \\
&>24\left(28 \pi-313+480 \times 0.7-160 \times(0.7)^{2}\right)=781.55 \cdots>0 .
\end{aligned}
$$

It follows from $(2.48)$ and $(2.49)$ that there exists $x_{2} \in(0.7,1)$ such that $\mu_{2}^{\prime}(x)$ is strictly decreasing on $\left(0.7, x_{2}\right]$ and strictly increasing on $\left[x_{2}, 1\right)$. This in conjunction with $(2.46)$ implies that there exists $x_{3} \in(0.7,1)$ such that $\mu_{2}(x)$ is strictly decreasing on $\left(0.7, x_{3}\right]$ and strictly increasing on $\left[x_{3}, 1\right)$. From (2.44) and the piecewise monotonicity of $\mu_{2}(x)$, we know that $\mu_{2}(x)<0$ for $x \in(0.7,1)$; this in conjunction with $(2.42)$ implies $\mu(x)<0$ for $x \in(0.7,1)$.

\section{Lemma 2.6 The function}

$$
\sigma(x)=\frac{\sqrt{1+x^{2}} \arctan ^{3} x-2(x-\arctan x)}{\left(1+x^{2}\right)^{2} \arctan ^{3} x}
$$

is strictly decreasing on $[0.55,1)$. Moreover, $\sigma(x)<0.236$ for $x \in[0.55,1)$.

Proof Differentiating $\sigma(x)$ yields

$$
\sigma^{\prime}(x)=\frac{\sigma_{1}(x)}{\left(1+x^{2}\right)^{3} \arctan ^{4} x}
$$

where

$$
\sigma_{1}(x)=6(x-\arctan x)+6 x^{2} \arctan x-8 x \arctan ^{2} x-3 x \sqrt{1+x^{2}} \arctan ^{4} x .
$$


From Lemma 2.5 and (2.51) we clearly see that

$$
\sigma_{1}(x)<6 x^{2} \arctan x-\frac{59}{10} x \arctan ^{2} x-3 x \arctan ^{4} x=x \arctan x \sigma_{2}(x)
$$

for $x \in[0.55,1)$, where

$$
\sigma_{2}(x)=6 x-\frac{59}{10} \arctan x-3 \arctan ^{3} x
$$

Differentiating $\sigma_{2}(x)$ leads to

$$
\begin{aligned}
& \sigma_{2}(0.55)=-0.0482086 \cdots, \quad \sigma_{2}(1)=-0.0872684 \cdots, \\
& \sigma_{2}^{\prime}(x)=\frac{\sigma_{3}(x)}{10\left(1+x^{2}\right)}, \\
& \sigma_{3}(x)=1+60 x^{2}-90 \arctan ^{2} x, \\
& \sigma_{3}(0.55)=-3.60662 \cdots, \quad \sigma_{3}(1)=5.48348 \cdots, \\
& \sigma_{3}^{\prime}(x)=\frac{60 \sigma_{4}(x)}{1+x^{2}}, \\
& \sigma_{4}(x)=2 x+2 x^{3}-3 \arctan x, \\
& \sigma_{4}(0.55)=-0.0757796 \cdots, \quad \sigma_{4}(1)=1.64381 \cdots, \\
& \sigma_{4}^{\prime}(x)=\frac{-1+8 x^{2}+6 x^{4}}{1+x^{2}}>0 .
\end{aligned}
$$

It follows from $(2.58)-(2.61)$ that there exists $x_{4} \in(0.55,1)$ such that $\sigma_{3}(x)$ is strictly decreasing on $\left(0.55, x_{4}\right]$ and strictly increasing on $\left[x_{4}, 1\right)$. This in conjunction with (2.55)(2.57) implies that there exists $x_{5} \in(0.55,1)$ such that $\sigma_{2}(x)$ is strictly decreasing on $\left(0.55, x_{5}\right]$ and strictly increasing on $\left[x_{5}, 1\right)$. Then from $(2.54)$ we clearly see that $\sigma_{2}(x)<0$ for $x \in(0.55,1)$.

Therefore, it follows from (2.50) and (2.52) that $\sigma(x)$ is strictly decreasing on $[0.55,1)$. Moreover, $\sigma(x) \leq \sigma(0.55)=0.235477 \cdots<0.236$ for $x \in[0.55,1)$.

\section{Lemma 2.7 The function}

$$
\kappa(x)=\frac{2\left(4+3 x^{2}\right) \sinh ^{-1}\left(x \sqrt{2+x^{2}}\right)-8 x \sqrt{2+x^{2}}}{\left(2+x^{2}\right)\left[\sinh ^{-1}\left(x \sqrt{2+x^{2}}\right)\right]^{3}}
$$

is strictly decreasing on $[0.55,1)$. Moreover, $\kappa(x)<0.771$ for $x \in[0.55,1)$.

Proof Simple computations lead to

$$
\begin{aligned}
& \kappa(0.55)=0.770758 \cdots \\
& \kappa^{\prime}(x)=\frac{8 \kappa_{1}(x)}{\left(2+x^{2}\right)^{2}\left[\sinh ^{-1}\left(x \sqrt{2+x^{2}}\right)\right]^{4}},
\end{aligned}
$$

where

$$
\kappa_{1}(x)=6 x\left(2+x^{2}\right)-3\left(2+x^{2}\right)^{3 / 2} \sinh ^{-1}\left(x \sqrt{2+x^{2}}\right)+x\left[\sinh ^{-1}\left(x \sqrt{2+x^{2}}\right)\right]^{2} .
$$


We claim that

$$
\sqrt{2} x-\frac{x^{3}}{6 \sqrt{2}}<\sinh ^{-1}\left(x \sqrt{2+x^{2}}\right)<\sqrt{2} x
$$

for $x \in(0,1)$. Indeed, let

$$
\begin{aligned}
& \eta_{1}(x)=\sinh ^{-1}\left(x \sqrt{2+x^{2}}\right)-\sqrt{2} x+\frac{x^{3}}{6 \sqrt{2}}, \\
& \eta_{2}(x)=\sinh ^{-1}\left(x \sqrt{2+x^{2}}\right)-\sqrt{2} x .
\end{aligned}
$$

Then we clearly see that

$$
\begin{aligned}
& \eta_{1}(0)=\eta_{2}(0)=0, \\
& \eta_{1}^{\prime}(x)=\frac{2}{\sqrt{2+x^{2}}}+\frac{\sqrt{2}}{4} x^{2}-\sqrt{2}, \\
& \eta_{2}^{\prime}(x)=\frac{2}{\sqrt{2+x^{2}}}-\sqrt{2}<0, \\
& \eta_{1}^{\prime}(0)=0, \\
& \eta_{1}^{\prime \prime}(x)=x\left(\frac{1}{\sqrt{2}}-\frac{2}{\left(2+x^{2}\right)^{3 / 2}}\right)>0 .
\end{aligned}
$$

Therefore, the double inequality (2.65) follows easily from (2.68)-(2.72).

Equation (2.64) and inequality (2.65) imply that

$$
\kappa_{1}(x)<6 x\left(2+x^{2}\right)-3\left(2+x^{2}\right)^{3 / 2}\left(\sqrt{2} x-\frac{x^{3}}{6 \sqrt{2}}\right)+x(\sqrt{2} x)^{2}=\frac{x}{4} \kappa_{2}(x),
$$

where

$$
\kappa_{2}(x)=16\left(3+2 x^{2}\right)-\sqrt{2}\left(12-x^{2}\right)\left(2+x^{2}\right)^{3 / 2} .
$$

Let $u=\sqrt{2+x^{2}}$, then $x^{2}=u^{2}-2, \sqrt{2}<u<\sqrt{3}$ and $\kappa_{2}(x)$ becomes

$$
\tilde{\kappa}(u)=-16+32 u^{2}-14 \sqrt{2} u^{3}+\sqrt{2} u^{5} .
$$

Equation (2.75) leads to

$$
\begin{aligned}
& \tilde{\kappa}(\sqrt{2})=0 \\
& \tilde{\kappa}^{\prime}(u)=u\left(64-42 \sqrt{2} u+5 \sqrt{2} u^{3}\right)=u \tilde{\kappa}_{1}(u), \\
& \tilde{\kappa}_{1}(u)=64-42 \sqrt{2} u+5 \sqrt{2} u^{3}, \quad \tilde{\kappa}_{1}(\sqrt{2})=0, \quad \tilde{\kappa}_{1}(\sqrt{3})=-2.1362 \cdots, \\
& \tilde{\kappa}_{1}^{\prime}(u)=15 \sqrt{2}\left(u-\sqrt{\frac{14}{5}}\right)\left(u+\sqrt{\frac{14}{5}}\right) .
\end{aligned}
$$

From (2.79) we clearly see that $\tilde{\kappa}_{1}^{\prime}(u)<0$ for $u \in(\sqrt{2}, \sqrt{14 / 5})$ and $\tilde{\kappa}_{1}^{\prime}(u)>0$ for $u \in$ $(\sqrt{14 / 5}, \sqrt{3})$. This in conjunction with (2.77) implies that $\tilde{\kappa}^{\prime}(u)$ is strictly decreasing on 
$(\sqrt{2}, \sqrt{14 / 5}]$ and strictly increasing on $[\sqrt{14 / 5}, \sqrt{3})$. Thus $\tilde{\kappa}^{\prime}(u)<0$ for $u \in(\sqrt{2}, \sqrt{3})$ follows from (2.78) and the piecewise monotonicity of $\tilde{\kappa}^{\prime}(u)$.

Therefore, $\kappa_{2}(x)=\tilde{\kappa}(u)<0$ follows from (2.76). This in conjunction with (2.63) and (2.73) implies that $\kappa(x)$ is strictly decreasing on $[0.55,1)$. Moreover, it follows from (2.62) that $\kappa(x) \leq \kappa(0.55)=0.770758 \cdots<0.771$ for $x \in[0.55,1)$.

Lemma 2.8 The function

$$
\tau(x)=\frac{2(x-\arctan x)}{\left(1+x^{2}\right)^{2} \arctan ^{3} x}-\frac{2 x\left(3+x^{2}\right)}{\left(2+x^{2}\right)^{3 / 2} \sinh ^{-1}\left(x \sqrt{2+x^{2}}\right)}<-0.88
$$

for $x \in[0.55,1)$.

Proof We first prove

$$
\sqrt{2+x^{2}} \sinh ^{-1}\left(x \sqrt{2+x^{2}}\right)<2 x+\frac{x^{3}}{3}
$$

for $x \in(0,1)$. Let

$$
\varepsilon(x)=\sqrt{2+x^{2}} \sinh ^{-1}\left(x \sqrt{2+x^{2}}\right)-\left(2 x+\frac{x^{3}}{3}\right) .
$$

Then $\varepsilon(x)<0$ follows from $\varepsilon(0)=0$ and the fact that

$$
\varepsilon^{\prime}(x)=\frac{x}{\sqrt{2+x^{2}}}\left(\sinh ^{-1}\left(x \sqrt{2+x^{2}}\right)-x \sqrt{2+x^{2}}\right)<\frac{x}{\sqrt{2+x^{2}}}\left(\sqrt{2} x-x \sqrt{2+x^{2}}\right)<0,
$$

where the second term follows from (2.65).

From Lemma 2.5 and (2.10) we clearly see that

$$
\frac{x-\arctan x}{\arctan ^{3} x}<\frac{7 x}{20 \arctan x}<\frac{21}{20\left(3-x^{2}\right)}
$$

for $x \in[0.55,1)$.

It follows from (2.80) and (2.81) that

$$
\tau(x)<\frac{21}{10\left(1+x^{2}\right)^{2}\left(3-x^{2}\right)}-\frac{6\left(3+x^{2}\right)}{\left(2+x^{2}\right)\left(6+x^{2}\right)}=: \tau_{1}(x)
$$

for $x \in[0.55,1)$.

Simple computation yields

$$
\begin{aligned}
& \tau_{1}(0.55)=-0.906585 \cdots, \quad \tau_{1}(1)=-0.880357 \cdots, \\
& \tau_{1}^{\prime}(x)=\frac{3 x}{5\left(x^{2}-3\right)^{2}\left(1+x^{2}\right)^{3}\left(2+x^{2}\right)^{2}\left(6+x^{2}\right)^{2}} \tilde{\tau}(x),
\end{aligned}
$$

where

$$
\begin{aligned}
\tilde{\tau}(x)= & -2,880+2,424 x^{2}+6,052 x^{4}+1,468 x^{6} \\
& -939 x^{8}-219 x^{10}+60 x^{12}+20 x^{14},
\end{aligned}
$$




$$
\begin{gathered}
\tilde{\tau}(0.55)=-1,560.68 \cdots, \quad \tilde{\tau}(1)=5,986 \\
\tilde{\tau}^{\prime}(x)=2 x\left(2,424+12,104 x^{2}+4,404 x^{4}-3,756 x^{6}\right. \\
\left.-1,095 x^{8}+360 x^{10}+140 x^{12}\right)>0 .
\end{gathered}
$$

From (2.85)-(2.87) we know that there exists $x_{6} \in(0.55,1)$ such that $\tilde{\tau}(x)<0$ for $x \in$ $\left(0.55, x_{6}\right)$ and $\tilde{\tau}(x)>0$ for $x \in\left(x_{6}, 1\right)$. This in conjunction with $(2.84)$ implies that $\tau_{1}(x)$ is strictly decreasing on $\left[0.55, x_{6}\right)$ and strictly increasing on $\left[x_{6}, 1\right)$.

Therefore, $\tau(x)<\tau_{1}(x) \leq \max \left\{\tau_{1}(0.55), \tau_{1}(1)\right\}=-0.880357 \cdots<-0.88$ follows from (2.83) and the piecewise monotonicity of $\tau_{1}(x)$.

\section{Main result}

Theorem 3.1 The double inequality

$$
\alpha Q(a, b)+(1-\alpha) T(a, b)<S_{C A}(a, b)<\beta Q(a, b)+(1-\beta) T(a, b)
$$

holds for all $a, b>0$ with $a \neq b$ if and only if $\alpha \leq 0$ and $\beta \geq \beta_{0}=\frac{\sqrt{3} \pi-4 \log (2+\sqrt{3})}{(\sqrt{2} \pi-4) \log (2+\sqrt{3})}=$ $0.29758 \cdots$.

Proof Since the Neuman mean $S_{C A}(a, b)$, the quadratic mean $Q(a, b)$ and the second Seiffert mean $T(a, b)$ are symmetric and homogeneous of degree 1 , without loss of generality, we assume that $a>b$. Let $v=(a-b) /(a+b) \in(0,1)$, then from (1.1)-(1.3) one has

$$
\begin{aligned}
& S_{C A}(a, b)=A(a, b) \frac{v \sqrt{2+v^{2}}}{\sinh ^{-1}\left(v \sqrt{2+v^{2}}\right)}, \\
& T(a, b)=A(a, b) \frac{v}{\arctan (v)}, \quad Q(a, b)=A(a, b) \sqrt{1+v^{2}} .
\end{aligned}
$$

Equations (3.2) and (3.3) lead to

$$
\frac{S_{C A}(a, b)-T(a, b)}{Q(a, b)-T(a, b)}=\frac{\frac{v \sqrt{2+v^{2}}}{\sinh ^{-1}\left(v \sqrt{2+v^{2}}\right)}-\frac{v}{\arctan (v)}}{\sqrt{1+v^{2}}-\frac{v}{\arctan (v)}} .
$$

It is easy to find that

$$
\begin{gathered}
\lim _{v \rightarrow 0^{+}} \frac{\frac{v \sqrt{2+v^{2}}}{\sinh ^{-1}\left(v \sqrt{2+v^{2}}\right)}-\frac{v}{\arctan (v)}}{\sqrt{1+v^{2}}-\frac{v}{\arctan (v)}}=0, \\
\lim _{v \rightarrow 1^{-}} \frac{\frac{v \sqrt{2+v^{2}}}{\sinh ^{-1}\left(v \sqrt{2+v^{2}}\right)}-\frac{v}{\arctan (v)}}{\sqrt{1+v^{2}}-\frac{v}{\arctan (v)}}=\beta_{0} .
\end{gathered}
$$

We investigate the difference between the convex combination of $Q(a, b), T(a, b)$ and $S_{C A}(a, b)$ as follows:

$$
\begin{aligned}
& p Q(a, b)+(1-p) T(a, b)-S_{C A}(a, b) \\
& \quad=A(a, b)\left[p \sqrt{1+v^{2}}+(1-p) \frac{v}{\arctan (v)}-\frac{v \sqrt{2+v^{2}}}{\sinh ^{-1}\left(v \sqrt{2+v^{2}}\right)}\right] .
\end{aligned}
$$


Let

$$
D_{p}(v)=p \sqrt{1+v^{2}}+(1-p) \frac{v}{\arctan (v)}-\frac{v \sqrt{2+v^{2}}}{\sinh ^{-1}\left(v \sqrt{2+v^{2}}\right)} .
$$

Then simple computations lead to

$$
\begin{aligned}
D_{p}\left(0^{+}\right)= & 0, \quad D_{p}\left(1^{-}\right)=p\left(\sqrt{2}-\frac{4}{\pi}\right)+\frac{4}{\pi}-\frac{\sqrt{3}}{\log (2+\sqrt{3})}, \quad D_{\beta_{0}}\left(1^{-}\right)=0 \\
D_{p}^{\prime}(v)= & p\left[\frac{v}{\sqrt{1+v^{2}}}+\frac{v}{\left(1+v^{2}\right) \arctan ^{2} v}-\frac{1}{\arctan v}\right]+\frac{v}{\left(\sinh ^{-1}\left(v \sqrt{2+v^{2}}\right)\right)^{2}} \\
& -\frac{1+v^{2}}{\sqrt{2+v^{2}} \sinh ^{-1}\left(v \sqrt{2+v^{2}}\right)}-\frac{v}{\left(1+v^{2}\right) \arctan ^{2} v}+\frac{1}{\arctan v} \\
D_{p}^{\prime \prime}(v)= & p \frac{\sqrt{1+v^{2}} \arctan ^{3} v-2(v-\arctan v)}{\left(1+v^{2}\right)^{2} \arctan ^{3} v} \\
& +\frac{2\left(4+3 v^{2}\right) \sinh ^{-1}\left(v \sqrt{2+v^{2}}\right)-8 v \sqrt{2+v^{2}}}{\left(2+v^{2}\right)\left(\sinh ^{-1}\left(v \sqrt{2+v^{2}}\right)\right)^{3}} \\
& +\frac{2\left(v-\arctan ^{2}\right)}{\left(1+v^{2}\right)^{2} \arctan ^{3} v}-\frac{2 v\left(3+v^{2}\right)}{\left(2+v^{2}\right)^{3 / 2} \sinh ^{-1}\left(v \sqrt{2+v^{2}}\right)} \\
= & p \sigma(v)+\kappa(v)+\tau(v),
\end{aligned}
$$

where $\sigma(x), \kappa(x)$ and $\tau(x)$ are defined as in Lemmas 2.6, 2.7 and 2.8, respectively.

From Lemmas 2.1-2.3 and (3.10) we clearly see that

$$
\begin{aligned}
D_{\beta_{0}}^{\prime}(v) & >\beta_{0}\left(\frac{v}{3}-\frac{v^{3}}{6}\right)-\frac{v}{3}+\frac{2 v^{3}}{45}-\frac{v^{5}}{63}+\frac{2 v}{3}-\frac{16 v^{3}}{45} \\
& =\frac{v}{630}\left[210\left(1+\beta_{0}\right)-7\left(28+15 \beta_{0}\right) v^{2}-10 v^{4}\right] \\
& >\frac{v}{630}\left[210(1+0.29758)-7(28+15 \times 0.29759) \times(0.55)^{2}-10 \times(0.55)^{4}\right] \\
& =\frac{v}{630} \times 202.83 \cdots>0
\end{aligned}
$$

for $v \in(0,0.55]$.

It follows from Lemmas 2.6-2.8 and (3.11) that

$$
D_{\beta_{0}}^{\prime \prime}(v)=\beta_{0} \sigma(v)+\kappa(v)+\tau(v)<0.236 \beta_{0}+0.771-0.88=-0.0387709 \cdots
$$

for $v \in[0.55,1)$. Then from $D_{\beta_{0}}^{\prime}(0.55)=0.0139552 \cdots$ and $D_{\beta_{0}}^{\prime}(1)=-0.0650268 \cdots$ we know that there exists $v_{0} \in(0.55,1)$ such that $D_{\beta_{0}}^{\prime}(v)>0$ for $v \in\left[0.55, v_{0}\right)$ and $D_{\beta_{0}}^{\prime}(x)<0$ for $v \in\left(v_{0}, 1\right)$. This in conjunction with (3.13) leads to the conclusion that $D_{\beta_{0}}(v)$ is strictly increasing on $\left[0.55, v_{0}\right]$ and strictly decreasing on $\left[v_{0}, 1\right)$.

Therefore, $D_{\beta_{0}}(v)>0$ for $v \in(0,1)$ follows from $(3.9)$ and the monotonicity of $D_{\beta_{0}}(v)$. In other words, we obtain

$$
\beta_{0} Q(a, b)+\left(1-\beta_{0}\right) T(a, b)>S_{C A}(a, b)
$$

for $a, b>0$ with $a \neq b$. 
Obviously, if $\alpha=0$, then (1.4) gives

$$
T(a, b)<S_{C A}(a, b)
$$

for $a, b>0$ with $a \neq b$.

Therefore, Theorem 3.1 follows from (3.14) and (3.15) together with the following statements:

- If $\alpha>0$, then (3.4) and (3.5) imply that there exists $\delta_{1} \in(0,1)$ such that $S_{C A}(a, b)<\alpha Q(a, b)+(1-\alpha) T(a, b)$ for all $a, b>0$ with $(a-b) /(a+b) \in\left(0, \delta_{1}\right)$.

- If $\beta<\beta_{0}$, then (3.4) and (3.6) imply that there exists $\delta_{2} \in(0,1)$ such that $S_{C A}(a, b)>\beta Q(a, b)+(1-\beta) T(a, b)$ for all $a, b>0$ with $(a-b) /(a+b) \in\left(1-\delta_{2}, 1\right)$.

\section{Competing interests}

The authors declare that they have no competing interests.

\section{Authors' contributions}

Y-MC provided the main idea and carried out the proof of Theorem 3.1. HW carried out the proof of Lemmas 2.1-2.4. T-HZ carried out the proof of Lemmas 2.5-2.8 and drafted the manuscript. All authors read and approved the final manuscript.

\section{Author details}

${ }^{1}$ School of Mathematics and Computation Science, Hunan City University, Yiyang, 413000, China. ${ }^{2}$ Department of Mathematics, Changsha University of Science and Technology, Changsha, 410077, China. ${ }^{3}$ Department of Mathematics, Hangzhou Normal University, Huangzhou, 311121, China.

\section{Acknowledgements}

The research was supported by the Natural Science Foundation of China under Grants 11301127 and 61374086, and the Natural Science Foundation of Zhejiang Province under Grant LY13A010004.

Received: 7 May 2014 Accepted: 14 July 2014 Published: 19 Aug 2014

\section{References}

1. Neuman, E: On some means derived from the Schwab-Borchardt mean. J. Math. Inequal. 8(1), 171-183 (2014)

2. Neuman, E: On some means derived from the Schwab-Borchardt mean II. J. Math. Inequal. 8(2), 361-370 (2014)

3. Neuman, E, Sándor, J: On the Schwab-Borchardt mean. Math. Pannon. 14(2), 253-266 (2003)

4. Neuman, E, Sándor, J: On the Schwab-Borchardt mean II. Math. Pannon. 17(1), 49-59 (2006)

5. Seiffert, H-J: Aufgabe $\beta$ 16. Ginkgo-Wurzel 29, 221-222 (1995)

6. Toader, G: Seiffert type means. Nieuw Arch. Wiskd. 17(3), 379-382 (1999)

7. Wang, M-K, Qiu, Y-F, Chu, Y-M: Sharp bounds for Seiffert means in terms of Lehmer means. J. Math. Inequal. 4(4), 581-586 (2010)

8. Zhao, T-H, Chu, Y-M, Liu, B-Y: Optimal bounds for Neuman-Sándor mean in terms of the convex combinations of harmonic, geometric, quadratic, and contraharmonic means. Abstr. Appl. Anal. 2012, Article ID 302635 (2012)

9. Jiang, W-D, Qi, F: Some sharp inequalities involving Seiffert and other means and their concise proofs. Math. Inequal. Appl. 15(4), 1007-1017 (2012)

10. Jiang, W-D: Some sharp inequalities involving reciprocals of the Seiffert and other means. J. Math. Inequal. 6(4), 593-599 (2012)

11. Neuman, E: A note on a certain bivariate mean. J. Math. Inequal. 6(4), 637-643 (2012)

12. Zhao, T-H, Chu, Y-M, Jiang, Y-L, Li, Y-M: Best possible bounds for Neuman-Sándor mean by the identric, quadratic and contraharmonic means. Abstr. Appl. Anal. 2013, Article ID 348326 (2013)

13. Sun, H, Song, Y-Q, Chu, Y-M: Optimal two parameter bounds for Seiffert mean. J. Appl. Math. 2013, Article ID 438971 (2013)

14. Zhang, Y, Chu, Y-M, Jiang, Y-L: Sharp geometric mean bounds for Neuman means. Abstr. Appl. Anal. 2014, Article ID 949815 (2014)

15. Neuman, E: On a new bivariate mean. Aequ. Math. (2013). doi:10.1007/s00010-013-0224-8

16. He, Z-Y, Chu, Y-M, Wang, M-K: Optimal bounds for Neuman means in terms of harmonic and contraharmonic means. J. Appl. Math. 2013, Article ID 807623 (2013)

17. Chu, Y-M, Qian, W-M: Refinements of bounds for Neuman means. Abstr. Appl. Anal. 2014, Article ID 354132 (2014)

18. Qian, W-M, Chu, Y-M: Optimal bounds for Neuman means in terms of geometric, arithmetic and quadratic means. J. Inequal. Appl. 2014, Article ID 175 (2014) 